\section{Statistical Mechanics}

Concepts in Statistical Mechanics. By Arthur Hobson. Pp. xii +172 . (Gordon and Breach: New York, London and Paris, January 1972.) £7.30; $\$ 17.50$.

Atoms and Atomic Theory: an Introduction to Statistical Mechanics. By Ralph Baierlein. Pp. xi+486. (W. H. Freeman: San Francisco, October 1971.) $\$ 15.00$; £6.60.

THEsE two volumes have one thing in common: they both base the discussion of statistical mechanics on Jaynes's view of the subject. This in turn was a development following from Shannon's statement of probability theory and information theory. There may be something to be said for this approach -which to some extent is nothing but a formalization of the point of view that statistical mechanics is the study of very incompletely specified systems -a point of view which is clear from the Ehrenfests' masterly account of statistical mechanics in the Enzyklopädie der Mathematischen Wissenschaften (1911) and which was most forcefully advanced by Tolman.

The book by Hobson is a pleasant account of the concepts and mathematical structure of statistical mechanics, well written and well thought out. One wonders, however, why this material is presented in book form. There is very little new material in the book and it certainly is not a textbook, so the reason for issuing it as a hardcover volume is completely obscure. I feel that the author would have been better advised to have submitted it for publication in a review journal, where it surely would reach a larger public.

The book by Baierlein is a textbook and the author has included a useful collection of problems at the end of each chapter. The main body of the book is straightforward conventional statistical mechanics, and one wonders why the title, blurb, and introduction stress the "alternative" approach. Moreover, if one stresses the information theory approach in a textbook, one should at least give an adequate description of other ways of introducing exactly the same theoretical framework for solving problems in the theory of complicated systems-and this is one of the strong points of Hobson's book.

The ground covered in Baierlein's book-after three chapters on probability theory-is also conventional; topics covered include equilibrium, paramagnetism, the classical limit, partition functions, perfect and imperfect gases, systems of identical particles (where, as so often in textbooks on statistical mechanics, the discussion of the connexion between spin and statis- tics is sloppy and incomplete), the free electron model of metals, Bose-Einstein condensation, black-body radiation, magnetic cooling, negative temperatures, and the density matrix. The discussion of these topics is on the whole thorough and sufficiently detailed for students. The book is a worthwhile addition to the already very large choice of texts on statistical mechanics. D. TER HAAR

\section{Geochemical Indicators}

Geochemical Facies Analysis. By Ernst Werner. (Methods in Geochemistry and Geophysics, Vol. 11.) Pp. vi 152. (Elsevier: Amsterdam, London and New York, 1970.) £3.50.

THIs book is an extended review which gives primary consideration to assessing the value and potential of those chemical elements and compounds which have already been widely accepted as geochemical indicators. The review draws on a substantial number of geochemical studies of facies which have been carried out during the past twenty-five years and, with its list of approximately 300 recent references, it should form a useful and relatively inexpensive source book for the student of sedimentary geochemistry.

The earlier part of the review is devoted chiefly to a discussion of factors which can affect the results of geochemical facies analysis; not only sampling, preparative and analytical methods, but also the broader influences on the composition of the sediment of climate, tectonism, diagenesis and metamorphism, sediment source and rate of sedimentation. Professor Werner finds that few, if any, of the definitions of facies so far proposed are satisfactory for geochemical purposes, either because they ignore geochemical data altogether, or because they are insufficiently refined. Instead, a special facies unit is proposed, the "hydrofacies", which refers to the influence of the physical and chemical composition of water on the sediments. The results of geochemical facies analysis are considered within the context of the three subdivisions of the hydrofacies that can be recognized-the salinity, oxygen and temperature facies. The reader is left in no doubt about the generally unsatisfactory nature and limited application of geochemical indicators within the salinity facies and it is acknowledged that the temperature facies is the most difficult subdivision of the hydrofacies to recognize. Studies which demonstrate the original oxygen distribution of the sediments are at present felt to be potentially the most useful and reliable, although, in the future, isotopes, and particularly organo-metallic complexes, are suggested as being the most promising environmental indicators.

Besides drawing together and analysing, albeit briefly, the results of a large number of investigations, an important contribution of the review is that it serves as a warning that no single chemical element or compound can be regarded as an infallible indicator of a specific depositional environment. The value of geochemistry is acknowledged, but more precise evaluations of the nature of environments are likely when geochemical data are combined with facies evidence from other geological sources.

Although there are some quaint usages of English in the review, generally the text reads easily. The two short chapters on non-chemical methods of facies analysis and the practical use and future importance of geochemical facies analysis could both have been profitably extended, particularly the former, but the lack of development of these sections does not detract significantly from a useful addition to this series on geochemical and geophysical methods.

D. G. MurCHISON

\section{Stellar Elements}

Nuclear Reactions in Stellar Surfaces and their Relations with Stellar Evolution. By Hubert Reeves. Pp. $\mathrm{x}+87$. (Gordon and Breach: New York, London and Paris. August 1971.) £4, $\$ 9.60$ cloth; $£ 1.95, \$ 4.75$ paperback.

THE existence of the light elements deuterium, lithium, beryllium and boron presents a special challenge to theories of nucleosynthesis in stars because the low stability of their nuclei makes one expect them to be largely destroyed under typical conditions in stellar interiors. The relative abundance of these elements in cosmic rays, however, provides a clue as to how they were probably formed, namely by spallation reactions between energetic protons and heavier nuclei in a rarefied medium (though other possibilities exist for D and ${ }^{7} \mathrm{Li}$ ). This general idea has received powerful support from a brilliant series of experimental and theoretical studies at the Institut de Physique Nucléaire, Orsay, in which the relevant cross-sections were measured and found to predict to a good approximation several of the elemental and isotopic abundance ratios of light elements observed in the solar system and a number of stars. Where the reactions that produced most of these elements actually occurred is another question. Originally the most likely location was thought to be in surface layers of the stars themselves, during flare activity such as is observed in the youthful $\mathrm{T}$ Tauri phase of evolution, but this hypothesis involves grave 